\title{
Photonic Generation of Square Waveform Based on Fiber Optical Parametric Oscillator
}

\author{
Sigang Yang, Chi Zhang, Yue Zhou, and Kenneth K. Y. Wong* \\ Photonics Systems Research Laboratory, Department of Electrical and Electronic Engineering, \\ The University of Hong Kong, Pokfulam Road, Hong Kong. \\ *E-mail: kywong@eee.hku.hk
}

\begin{abstract}
We demonstrate photonic generation of square waveform using fiber optical parametric oscillator without the external signal source. Stable square waveform pulse train at the repetition rate of $1.133 \mathrm{GHz}$ is achieved.

(C)2010 Optical Society of America

OCIS codes: (060.4370) Nonlinear optics, fibers; (190.4970) Parametric oscillators and amplifiers
\end{abstract}

\section{Introduction}

Parametric oscillators based on fiber optical parametric amplification (FOPA) have attracted significant interests recently [1]. Pulsed pump fiber optical parametric oscillators (FOPOs) can provide remarkably wavelength span as large as $500 \mathrm{~nm}$ [2]. Nevertheless, it is required to have delicate picosecond pulse source. Continuous-wave (CW) pump FOPOs are also investigated [3]. A main problem with CW pump FOPO is that the cavity length is usually long, so that multiple longitudinal modes oscillate simultaneously. Under ordinary circumstances, phases of these multiple longitudinal modes have random relationships. In order to overcome this problem, we have previously proposed an actively mode-locked FOPO to lock the phase of the longitudinal modes [4]. However, the modulation frequency must be the precise harmonics of the free spectral range (FSR) of the FOPO cavity. The cavity easily loses synchronism with the modulation signal because it would readily drift due to the temperature variation or environmental disturbance.

In this paper, we propose a novel mode-locked FOPO. It simply relies on the beating radio frequency (RF) signal of the longitudinal modes to drive the intracavity amplitude modulator. The cavity is synchronized with the RF driving signal automatically. Due to the long cavity length of the FOPO, we use two subring cavities with short cavity length to enlarge the effective longitudinal mode spacing, and increase the modulation frequency at the same time. The FOPO can produce stable square waveform pulse train at the repetition rate of $1.133 \mathrm{GHz}$ without any external RF signal source. The tuning range is over $10 \mathrm{~nm}$, which is only limited by the FOPA gain bandwidth under the current CW pump configuration.

\section{Experimental Setup}

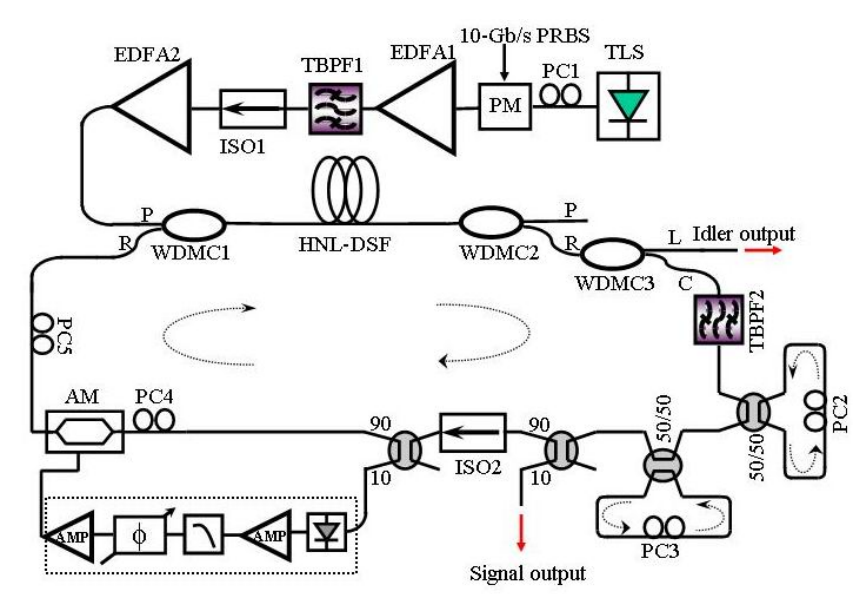

Fig. 1. Schematic diagram of the square waveform generator based on FOPO.

The schematic diagram of the proposed FOPO is shown in Fig. 1. The pump was seeded by an external cavity tunable laser source (TLS). In order to suppress stimulated Brillouin scattering (SBS), the light was first phase-modulated with 10-Gbps pseudo-random bit sequence (PRBS) signal via a phase modulator (PM). The SBS 


\section{JTuD82.pdf}

could be suppressed by up to $28 \mathrm{~dB}$. Then the pump was amplified by a two-stage configuration of EDFAs, with a maximum average output power of $33 \mathrm{dBm}$. After that the amplified pump was coupled into a 400-m highly-nonlinear dispersion-shifted fiber (HNL-DSF) via the P-port $(1554.89 \sim 1563.89 \mathrm{~nm})$ of a bandpass wavelength-divisionmultiplexing (WDM) coupler (WDMC1). The high power pump propagated through the HNL-DSF and then was rejected via the P-port of another bandpass WDM coupler (WDMC2), while the amplified signal and idler were propagated through the R-port $(1500 \sim 1551 \mathrm{~nm}, 1567 \sim 1620 \mathrm{~nm})$ of WDMC2. A C/L band WDM coupler was then inserted to allow only the $\mathrm{C}$ band signal to circulate in the cavity. The signal from the C-port of WDMC3 was filtered by a $0.8-\mathrm{nm}$ band pass filter (TBPF2). Two sub-ring cavities with different cavity length of $6.2 \mathrm{~m}$ and $6.5 \mathrm{~m}$ respectively were then inserted after TBPF2. The FOPO provided the output through a 10/90 output coupler. Another 10/90 coupler was used to provide the feedback. This feedback loop consisted of a photodetector (PD) with 12-GHz bandwidth, a $7.5 \mathrm{GHz}$ low pass filter, a variable electrical phase shifter denoted by $\phi$, and RF amplifiers (AMPs). A $\mathrm{LiNbO}_{3}$ Mach-Zehnder amplitude modulator (MZ-AM) driven by the feedback signal was inserted. PC5 was used to align the signal's state of polarization (SOP) with that of the pump so as to maximize the signal gain.

\section{Results and Discussions}

The proposed FOPO simply utilizes the beating RF signal of the longitudinal modes to drive the MZ-AM. Due to the introduction of the two short subring cavities, the effective longitudinal mode spacing is broadened to be $1.133 \mathrm{GHz}$. The driving RF signal is synchronized with the cavity automatically. Fig. 2(a) shows the measured RF spectrum of the output signal light pulse train. The fundamental frequency and the odd harmonics can be observed while the even harmonics are prohibited. Fig. 2(b) shows the generated optical pulse trains observed on a digital communication analyzer (DCA). It demonstrates that the square waveform pulse train at the repetition rate of $1.133 \mathrm{GHz}$ is produced.

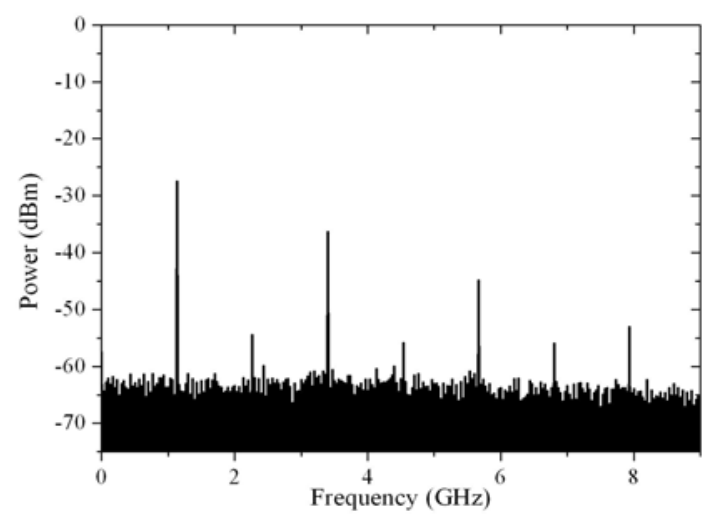

(a)

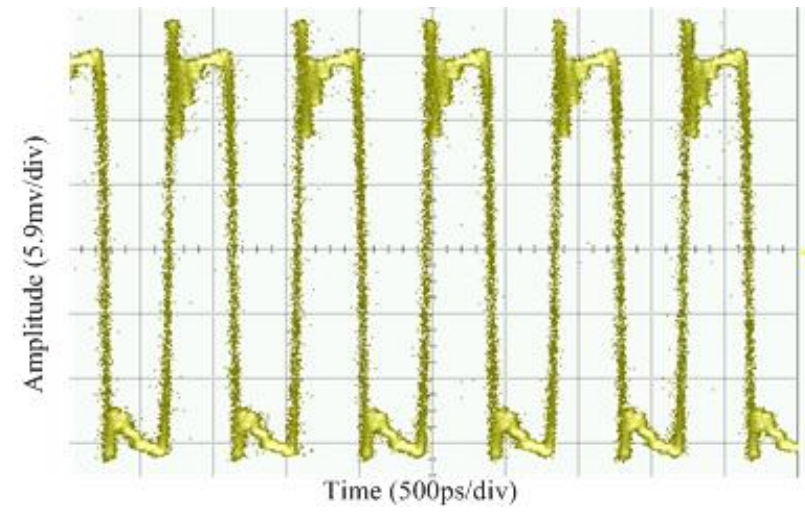

(b)

Fig. 2. (a) Measured RF spectrum of the output signal light pulse train. (b) Measured waveform of the output signal light .

\section{Conclusion}

We have demonstrated photonic generation of the square waveform using FOPO. The FOPO works fundamentally by extracting the beating RF signal of the longitudinal modes to drive the intracavity amplitude modulator. In order to broaden the longitudinal mode spacing, two subring cavities with short cavity length are inserted. Without any external RF signal source, the FOPO can output stable square waveform pulse train at the repetition rate of $1.133 \mathrm{GHz}$

\section{Acknowledgment}

The work was partially supported by grants from the research Grants Council of the Hong Kong Special Administrative Region, China (Project No. HKU7179/08E and HKU7183/09E). The authors would also like to thank Sumitomo Electric Industries for providing HNL-DSF.

\section{References}

[1] J. E. Sharping et al. "Microstructure Fiber Based Optical Parametric Oscillators", IEEE J. Lightwave Technol., 26, 2184-2191(2008).

[2] Y. Zhou et al. "Widely-tunable picosecond optical parametric oscillator using highly-nonlinear fiber", Opt Lett., 34,989 - 991(2009).

[3] M. E. Marhic et al. "Continuous-wave fiber optical parametric oscillator", Opt. Lett., 27, 1439 - 1441(2002).

[4] S. Yang et al. "Actively Mode-Locked Fiber Optical Parametric Oscillator", IEEE J. Select. Topics Quant. Electron., 15, 393-398(2009). 